\title{
Synthesis of Boron Nitride Nanotubes by Self-Propagation High-Temperature Synthesis and Annealing Method
}

\author{
Jilin Wang, ${ }^{1}$ Yunle Gu, ${ }^{1,2}$ Laiping Zhang, ${ }^{1}$ Guowei Zhao, ${ }^{1}$ and Zhanhui Zhang ${ }^{1,2}$ \\ ${ }^{1}$ School of Materials Science and Engineering, Wuhan Institute of Technology, Wuhan 430073, China \\ ${ }^{2}$ Nano and Ceramic Materials Research Center, Wuhan Institute of Technology, Wuhan 430073, China \\ Correspondence should be addressed to Yunle Gu, ncm@mail.wit.edu.cn
}

Received 30 September 2010; Accepted 29 December 2010

Academic Editor: Edward Andrew Payzant

Copyright (c) 2010 Jilin Wang et al. This is an open access article distributed under the Creative Commons Attribution License, which permits unrestricted use, distribution, and reproduction in any medium, provided the original work is properly cited.

\begin{abstract}
High-quality boron nitride nanotubes were synthesized by annealing porous precursor in flowing $\mathrm{NH}_{3}$ gas at $1150^{\circ} \mathrm{C}$. The porous precursor $\mathrm{B}_{18} \mathrm{Ca}_{2}(\mathrm{MgO})_{9}$ was produced by self-propagation high-temperature synthesis ( $\mathrm{SHS}$ ) method using $\mathrm{Mg}, \mathrm{B}_{2} \mathrm{O}_{3}$, and $\mathrm{CaB}_{6}$ as the starting materials, which played an important role in synthesis of BN nanotubes in large quantities. Samples were characterized by SEM, TEM, EDX, HRTEM, X-ray powder diffraction (XRD), Raman, and Fourier transform infrared (FTIR) spectroscopy. The as-synthesized BN nanotubes have an average diameter of about $150 \mathrm{~nm}$ with a wall/diameter ratio of 2/3. Mean length of the BN nanotubes was more than $10 \mu \mathrm{m}$. The effects of temperature, time, and the possible mechanism of the growth of the $\mathrm{BN}$ nanotubes were also discussed.
\end{abstract}

\section{Introduction}

Boron nitride (BN) nanotubes have a similar structure as carbon nanotubes (CNTs). They also exhibit some attracting properties, including a stable insulator, a superresistance to oxidation at high temperatures, an excellent thermal conductivity, uniform electronic properties, interesting piezoelectricity, and optical properties [1]. Moreover, in addition to these characteristics, $\mathrm{BN}$ nanotubes also can be used as a perfect insulating tubular shield to encapsulate some materials within and demonstrated distinctive features [2-4]. Obviously, the unique properties of BN nanotubes will be the prerequisite of the important applications in the fields of optoelectronics, nanosemiconductor devices, electronics, energy storage, and biomedical medicine [5].

In recent years, various synthetic methods have been used to grow BN nanotubes. Generally, it could be classified into two categories, the high-temperature (above $2400^{\circ} \mathrm{C}$ ) method and the low-temperature (ranging from 400 to $1700^{\circ} \mathrm{C}$ ) method [6]. The high-temperature methods usually need special sophisticated instruments or complex processes to meet the high-temperature requirements and only obtain single morphology $\mathrm{BN}$ nanotubes, which was unaffordable.
In contrast to the high-temperature methods, the lowtemperature methods can operate at a mild condition and normal pressure. Importantly, different types of $\mathrm{BN}$ nanotubes could also be prepared by changing annealing conditions, which may be suitable for popularization and application. And the catalytic chemical vapor deposition (CVD) method is one of the most popular techniques for the low-temperature synthesis of BN nanotubes. Various kinds of boron-containing precursors can be used to prepare $\mathrm{BN}$ nanotubes. Commonly, the precursors can be diborane $\left(\mathrm{B}_{2} \mathrm{H}_{6}\right)[7,8]$, borazine $\left(\mathrm{B}_{3} \mathrm{~N}_{3} \mathrm{H}_{6}\right)[9,10]$ and trimethyl bo rate $\left(\mathrm{C}_{3} \mathrm{H}_{9} \mathrm{BO}_{3}\right)$ [11], elemental boron [12-18], iron boride $(\mathrm{FeB})[19,20]$, and boric acid $\left(\mathrm{H}_{3} \mathrm{BO}_{3}\right)[21]$. In addition, for the CVD method, precursors with uniform dispersion of metal catalyst and boron source are especially significant. Many efforts have been made to deal with the problem, such as ball-milling process [22-27], which is very consumptive of energy and time.

Herein, we introduce a convenient way so-called SHSannealing method to synthesize $\mathrm{BN}$ nanotubes using an effective porous precursor. The porous precursor can be readily produced by self-propagation high-temperature synthesis (SHS), which plays an important role in synthesis of 


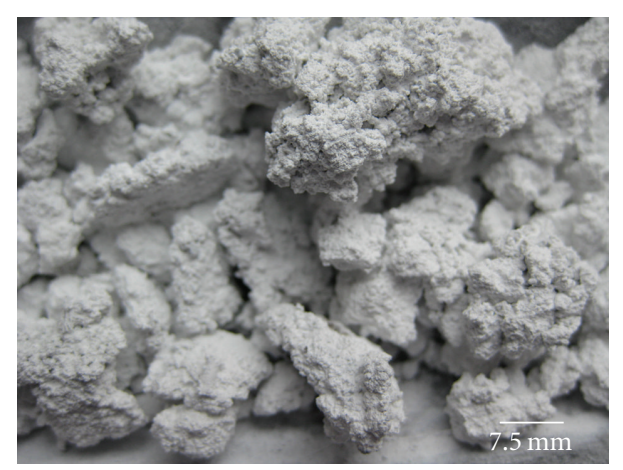

FIgure 1: Photo of the crude product in an alumina boat. Scale bar: $7.5 \mathrm{~mm}$.

large quantities of BN nanotubes. The effects of temperature, time, and possible mechanism of the growth of the $\mathrm{BN}$ nanotubes were also discussed.

\section{Experimental}

2.1. Materials. The reagents, magnesium $(\mathrm{Mg})$, and boron oxide $\left(\mathrm{B}_{2} \mathrm{O}_{3}\right)$ were of analytical pure grade and about 150 300 mesh $(50-100 \mu \mathrm{m})$ powders. Calcium hexaboride $\left(\mathrm{CaB}_{6}\right)$ was made by ourselves having the particle size of 0.2 to $0.95 \mu \mathrm{m}$ and the purity of $\geq 95 \mathrm{wt} \%$.

2.2. Instrumentation. The resultant $\mathrm{BN}$ nanotube samples were characterized by X-ray powder diffraction (XRD) using a Shimadzu XD-5A X-ray diffractometer using $\mathrm{Cu}$ $\mathrm{K} \alpha$ radiation (wavelength $\lambda=1.5406 \AA$ ). Raman spectrum was recorded at room temperature on a Nicolet DXR Raman spectrometer using Nd:YAG laser at excitation of $532 \mathrm{~nm}$. Fourier transform infrared (FTIR) spectra were recorded on a Nicolet 6700 Fourier transform infrared spectrometer in transmission mode using a $\mathrm{KBr}$ wafer. The scanning electron microscopy (SEM) was studied using a Hitachi S$3400 \mathrm{~N}$ scanning electron microscope and energy-dispersive X-ray (EDX) spectroscopy attached to it was employed to determine chemical contents. Samples were observed by directly spreading the samples on conductive tapes and sputtering $\mathrm{Au}$ or $\mathrm{Pt}$ on the surface. Transmission electron microscopy (TEM) and high-resolution transmission electron microscopy (HRTEM) were performed on a JEOL JEM2100F transmission electron microscope. For HRTEM and TEM observations, samples were prepared by ultrasonically dispersing the product in ethanol and depositing on copper grids coated with carbon film.

2.3. Synthesis of Boron Nitride Nanotubes by SHS-Annealing Method. In a typical experimental procedure, $11.90 \mathrm{~g} \mathrm{~B}_{2} \mathrm{O}_{3}$, $12.20 \mathrm{~g} \mathrm{Mg}$, and $11.60 \mathrm{~g} \mathrm{CaB}_{6}$ were mixed by a blender mixer for about 12 minutes. The mixture was put into a SHS-furnace and heated to $750^{\circ} \mathrm{C}$ for 10 minutes in an argon atmosphere. After the SHS reaction, $34.80 \mathrm{~g}$ porous precursor was obtained. The porous precursor was placed in an alumina boat and heated to $1150^{\circ} \mathrm{C}$ at $6^{\circ} \mathrm{C} / \mathrm{min}$ and kept for $6 \mathrm{~h}$ in an ammonia flowing gas at $0.3-0.9 \mathrm{~L} / \mathrm{min}$ in the center of a horizontal tubular furnace at a normal pressure. After cooled to room temperature naturally, the furnace was opened, and the crude product (shown in Figure 1) was collected and washed with $5 \mathrm{M}$ hydrochloric acid, ethanol, and distilled water. Finally, 20.25 g gray BN nanotube powders were obtained by drying in vacuum at $80^{\circ} \mathrm{C}$ for 24 hours. The yields of $\mathrm{BN}$ nanotubes based on boron were about $83.08 \mathrm{wt} \%$.

\section{Results and Discussion}

3.1. Results. The typical SEM image of as-synthesized BN nanotubes is shown in Figure 2(a), which indicates the products possess a high density of one-dimensional structures with diameters in the range of 30-200 $\mathrm{nm}$ and an average of about $150 \mathrm{~nm}$. The average lengths of the structures are more than $10 \mu \mathrm{m}$. Few BN flakes and particles were observed. The purity of BN nanotubes was estimated of about $95 \mathrm{wt} \%$.

Figures 2(b) and 2(c) give the typical TEM images of BN nanotubes with different diameters. The thin $\mathrm{BN}$ nanotube reveals a diameter of $60 \mathrm{~nm}$ with the wall thickness of $20 \mathrm{~nm}$ (Figure 2(b)). However, the thick BN nanotube presents a diameter of $180 \mathrm{~nm}$ with the wall thickness of $60 \mathrm{~nm}$, as seen in Figure 2(c). In addition, both the thin and the thick BN nanotubes had a wall/diameter rate of approximately $2 / 3$.

To investigate the structural features of the synthesized BN nanotubes, HRTEM observation was carried out. The typical HRTEM image of the BN nanotubes suggests a wellcrystallized structure, with outerwalls that are very clean (no amorphous BN coatings), as shown in Figure 2(d). The boron nitride nanotube has multiple walls with thickness of about $60 \mathrm{~nm}$ and clearly exhibit fringes with an average interlayer distance about $0.34 \mathrm{~nm}$ (see insert image of partial enlargement denoted by a frame in Figure 2(d)), which corresponds to the (002) plane of hexagonal BN crystal.

The EDX spectrum presented in Figure 2(e) demonstrates corresponding signals of boron and nitrogen, and quantitative analysis indicates that the molar ratio of $\mathrm{B}: \mathrm{N}$ is $1: 1.046$, which is close to that of $\mathrm{BN}$. The $\mathrm{O}$ peak can be ascribed to the slight surface oxidation of $\mathrm{BN}$ nanotubes. It is worth noting that the Ca signal also was revealed in the EDX spectrum, while no signal of $\mathrm{Mg}$ was observed. Probably, the Ca particles were encapsulated at the root of $\mathrm{BN}$ nanotubes as the metallic catalyst during the $\mathrm{BN}$ nanotubes growth process. A small amount of metal Ca particles still remained after the annealed precursor was washed with $5 \mathrm{M}$ hydrochloric acid. On the contrary, the $\mathrm{MgO}$ particles only acted as a supporter during the BN nanotubes growth process and were removed after the purification processes mentioned above.

Figure 3 displays the typical XRD pattern of the assynthesized $\mathrm{BN}$ nanotubes. Four peaks at $d$-spacings of 3.35, $2.17,2.07,1.67$, and $1.25 \AA$ can be indexed as (002), (100), (101), (004), and (110) planes of hexagonal boron nitride. The lattice constants are $a=2.509 \AA$ and $c=6.691 \AA$, close to the reported value $a=2.502$ and $c=6.660 \AA$ in JCPDF card no. 45-0893, indicating the good crystallinity of the $\mathrm{BN}$ 


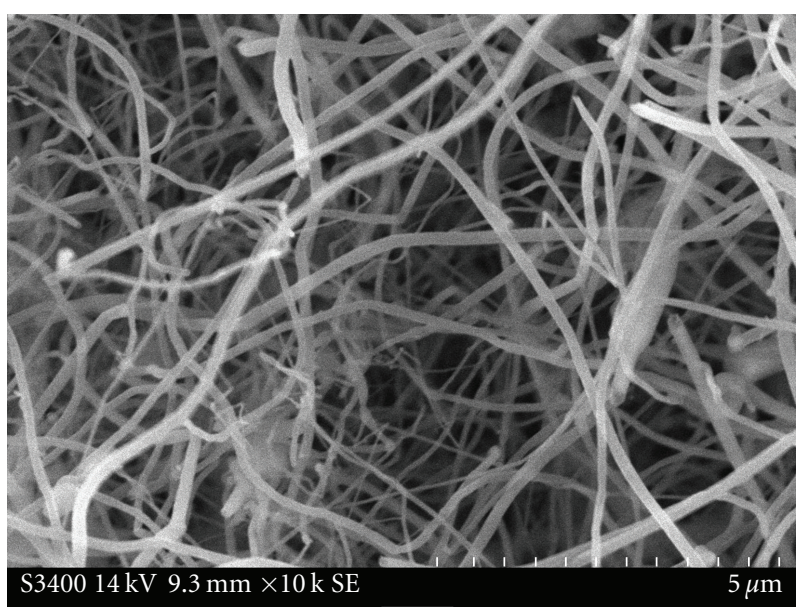

(a)

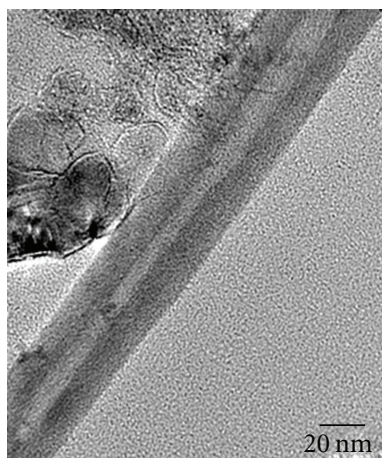

(b)

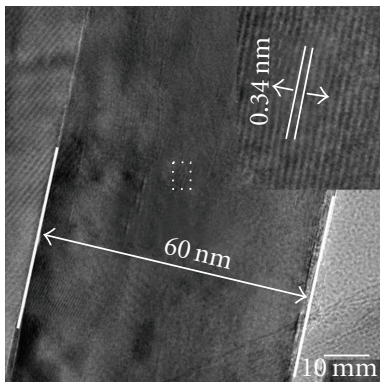

(d)

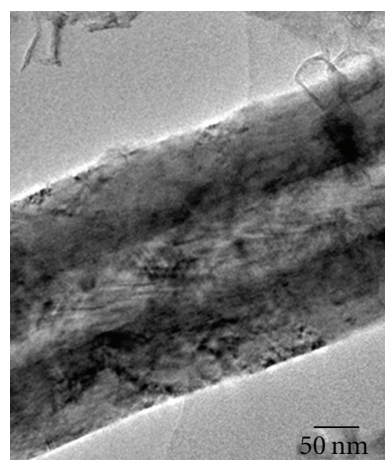

(c)

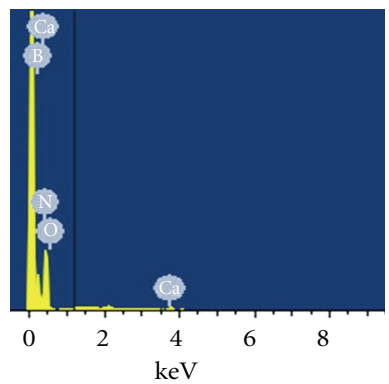

(e)

Figure 2: Typical SEM (a), TEM (b, c), HRTEM (d) images, and EDX spectrum (e) of the as-synthesized BN nanotubes. Scale bars: (a) $5 \mu$ m, (b) $20 \mathrm{~nm}$, (c) $50 \mathrm{~nm}$, and (d) $10 \mathrm{~nm}$.

nanotubes. No noticeable peaks of other impurities, such as $\mathrm{MgO}$ and $\mathrm{B}_{2} \mathrm{O}_{3}$, were detected in this pattern.

Figure 4(a) shows the typical wide-scan FTIR spectrum in the range of 500 to $4000 \mathrm{~cm}^{-1}$ of the as-synthesized BN nanotubes sample. Two strong peaks located at 803 and $1379 \mathrm{~cm}^{-1}$ can be ascribed to the out-of-plane B-N TO models of the $\mathrm{sp}^{2}$-bonded $\mathrm{h}-\mathrm{BN}$ and the $\mathrm{B}-\mathrm{N}-\mathrm{B}$ inplane bonding vibrations, respectively. The broad absorption band near $3454 \mathrm{~cm}^{-1}$ can be resulted from the O$\mathrm{H}$ bonds due to the absorbed of water. As shown in Figure 4(b), a peak at $1535 \mathrm{~cm}^{-1}$ in the deconvolution spectrum should be assigned to the unique stretching of the h-BN network around the circumference of $\mathrm{BN}$ nanotubes [28], while the other peak at $1117 \mathrm{~cm}^{-1}$ in the deconvolution spectrum may be attributed to the abnormal vibrations like those of wurtzite $\mathrm{BN}$, which may exist due to structure defects in the as-synthesized cylindrical $\mathrm{BN}$ nanotubes.

Figure 5 is the typical Raman spectrum of the assynthesized BN nanotubes. A sharp Raman peak was showed at around $1354 \mathrm{~cm}^{-1}$, which corresponded to the $E_{2 \mathrm{~g}}$ inplane model of the h-BN networks [29], while the other weak peak at about $780 \mathrm{~cm}^{-1}$ may be due to the glass holder used during the analysis. 


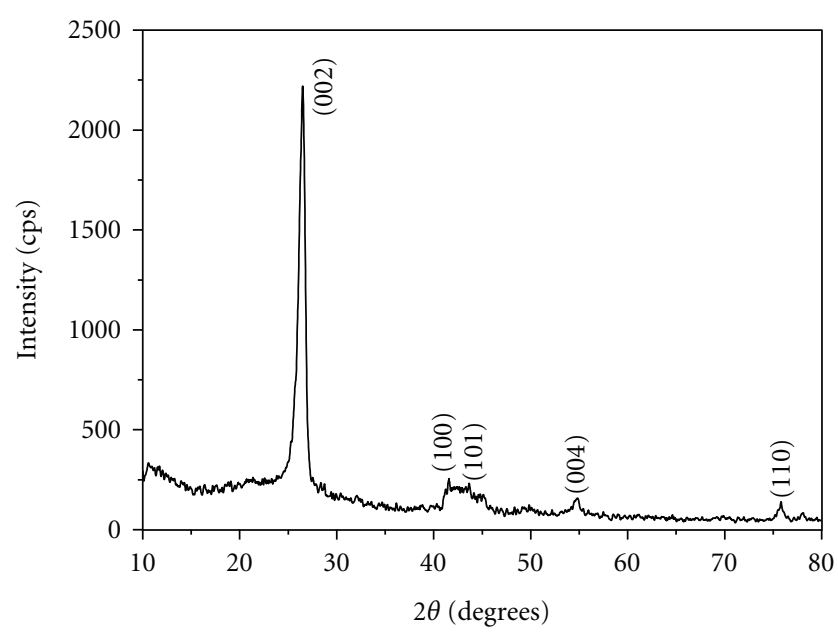

FIGURE 3: Typical XRD pattern of as-synthesized BN nanotubes.

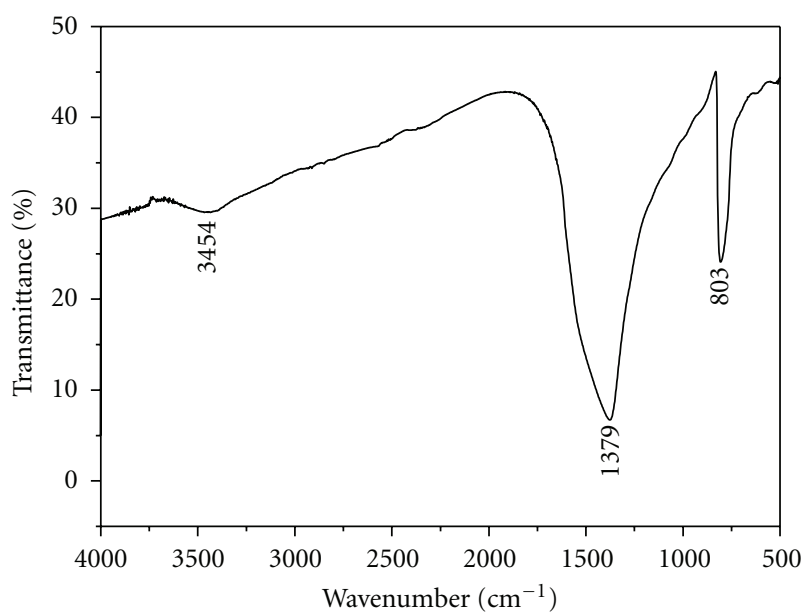

(a)

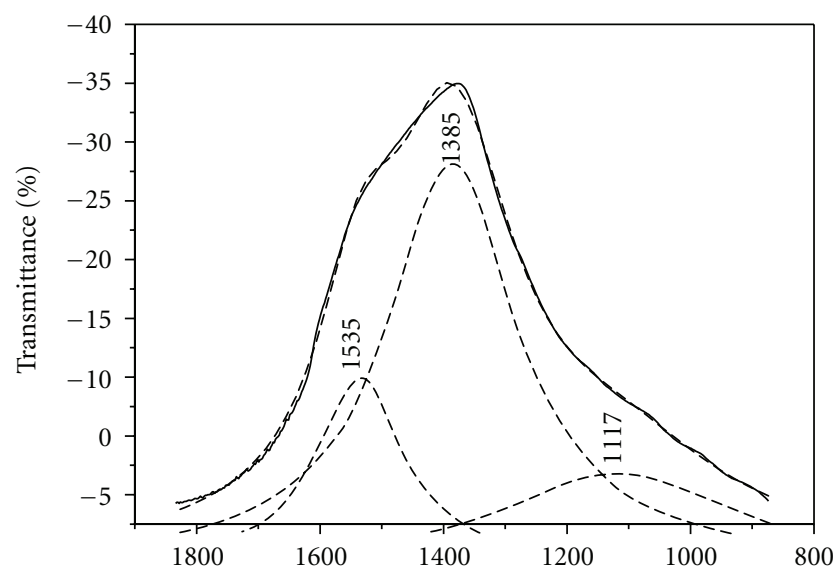

(b)

FIgUre 4: Typical FTIR and deconvolution FTIR spectra of the as-synthesized BN nanotubes. (a) Wide-scan FTIR spectrum and (b) deconvolution FTIR Spectrum.

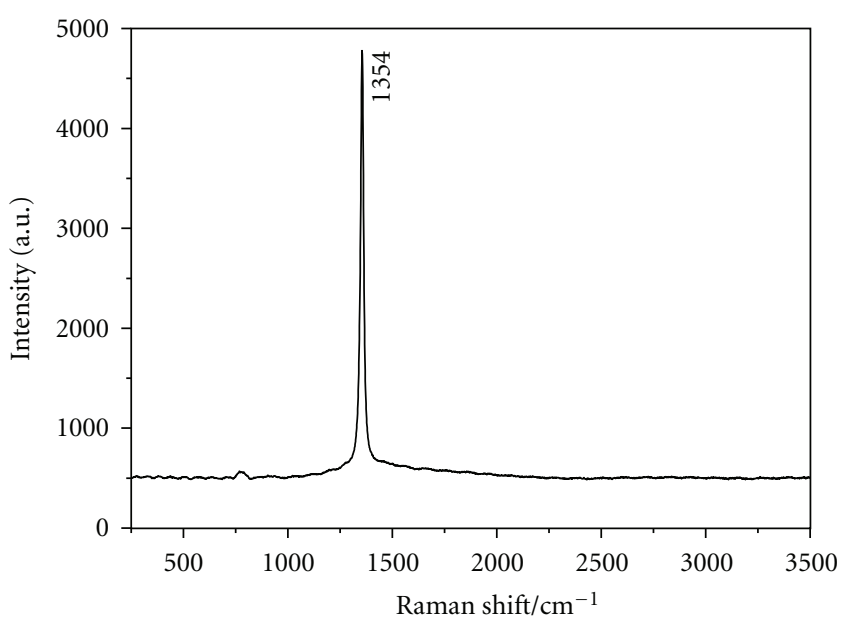

FIGURE 5: Typical Raman spectrum of the as-synthesized BN nanotubes.

3.2. Reaction Mechanism. In this experiment, the possible reactions are likely to occur as follows:

$$
\begin{aligned}
& 3 \mathrm{~B}_{2} \mathrm{O}_{3}+9 \mathrm{Mg}+2 \mathrm{CaB}_{6}=\mathrm{B}_{18} \mathrm{Ca}_{2}(\mathrm{MgO})_{9}, \\
& \mathrm{~B}_{18} \mathrm{Ca}_{2}(\mathrm{MgO})_{9}+18 \mathrm{NH}_{3} \\
& \quad=18 \mathrm{~B}^{*}+18 \mathrm{~N}^{*}+\mathrm{Ca}_{2}(\mathrm{MgO})_{9}+27 \mathrm{H}_{2}, \\
& x\left(\mathrm{~B}^{*}+\mathrm{N}^{*}\right)+\mathrm{Ca}_{2}(\mathrm{MgO})_{9}=x \mathrm{BN}_{\mathrm{NT}}+2 \mathrm{Ca}+9 \mathrm{MgO} \\
& (18-x)\left(\mathrm{B}^{*}+\mathrm{N}^{*}\right) \\
& =(18-x) \mathrm{BN}_{\mathrm{FL}}(x=0 \text { to } 18, \mathrm{NT}=\text { nanotubes, } \mathrm{FL}=\text { flakes }) .
\end{aligned}
$$

$\mathrm{B}_{18} \mathrm{Ca}_{2}(\mathrm{MgO})_{9}$ only stands for the mixed chemical elements in the precursor prepared by SHS reaction using $\mathrm{Mg}, \mathrm{B}_{2} \mathrm{O}_{3}$, and $\mathrm{CaB}_{6}$ as the starting materials, as in (1). $\mathrm{NH}_{3}$ reacted with $\mathrm{B}_{18} \mathrm{Ca}_{2}(\mathrm{MgO})_{9}$ at the temperature of $1150^{\circ} \mathrm{C}$ and produced chemically active $\mathrm{B}^{*}$ and $\mathrm{N}^{*}$ vapor and hydrogen gas, as in (2). According to the VLS growth mechanism [30], boron nitride nanotubes began to grow on the surface of metal catalyst liquid supported on $\mathrm{MgO}$, as in (3). However, flakes of BN may be synthesized due to lacking of metallic catalyst as vapor-solid (VS) growth mechanism, and the nanotube selectivity was $x / 18$, as in (4), where the values of $x$ were approximately estimated as about 16 to 18 .

3.3. Growth Mechanism of Boron Nitride Nanotubes. According to the catalytic VLS growth mechanism [30], the diameters of nanotubes depend on the particle sizes of catalyst liquid drops. Small droplets could lead to BN nanotubes with small diameters, while large droplets could render large diameters. Therefore, the distribution of catalyst drop sizes leads to a certain diameter range of $\mathrm{BN}$ nanotubes. Moreover, to grow a cylindrical $\mathrm{BN}$ nanotube by the base growth mechanism, there is at least one initial open tip. 


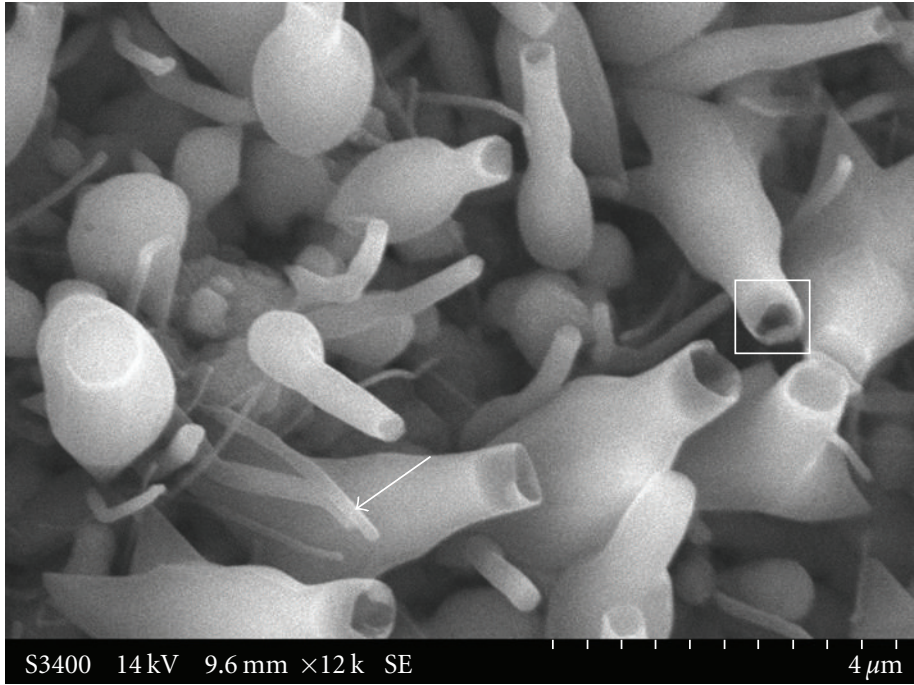

(a)

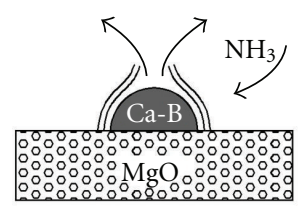

(b)

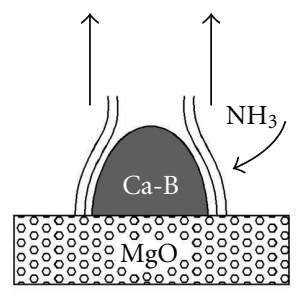

(c)

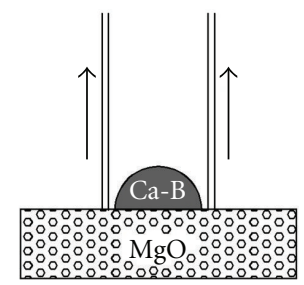

(d)

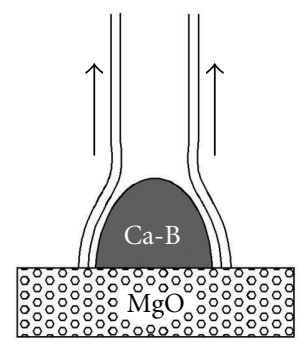

(e)

FIGURE 6: Typical SEM image of the porous SHS precursor at the initial annealing stage (a) and schematic illustration of base growth model of BN nanotubes with small (b) and large (c) diameters. Scale bars: (a) $4 \mu \mathrm{m}$.

The growth rate can be either approximately a constant or smoothly changing due to the change of microcircumstance such as the vapor constitution, temperature, and catalyst. The proposed growth model agrees well with the observed morphology of the as-synthesized BN nanotubes.

Figure 6(a) shows the typical SEM image of the porous SHS precursor at the initial annealing stage. It can be observed that there were many $\mathrm{BN}$ nanotube embryos of small (pointed by an arrow) and large (in a frame) sizes. Interestingly, all of these embryos revealed open tips. Therefore, we suggest a base growth scenario as schematically illustrated in Figures 6(b)-6(e).

Firstly, the Ca-B liquid drop is formed supported on $\mathrm{MgO}$ particle and reacts with $\mathrm{NH}_{3}$ gas at a high temperature $\left(1150^{\circ} \mathrm{C}\right)$. Secondly, when the concentrations of B and $\mathrm{N}$ species are supersaturated, $\mathrm{BN}$ nuclei begin to precipitate on the surface of Ca-B liquid drop and form a top-opened BN cap (Figures 6(b) and 6(c)). Thirdly, with the continuous supply of $\mathrm{B}$ and $\mathrm{N}$ atoms, the cap grows gradually up into a cylindrical BN nanotube with an open tip (Figures 6(d) and $6(\mathrm{e}))$. The growth of $\mathrm{BN}$ nanotube will not be terminated unless the $\mathrm{B}$ or $\mathrm{N}$ atoms are consumed. In this base growth process, $\mathrm{BN}$ nanotube grows from the liquid-solid interface in order to give the lowest liquid-solid interfacial energy [31].

3.4. Contrast Experiments. A collection of experiments were carried out to investigate the influence of the reaction temperature, time, and starting materials on the growth of $\mathrm{BN}$ nanotubes.

It was found that the optimum temperature range for the growth of the as-synthesized $\mathrm{BN}$ nanotubes was in the range of $1050-1150^{\circ} \mathrm{C}$. The crystallinity of $\mathrm{BN}$ nanotubes will become better with the rise of temperature in this range. If the reaction temperature is lower than $1050^{\circ} \mathrm{C}$, both selectivity and the yield of $\mathrm{BN}$ nanotubes will decrease. Temperature above $1150^{\circ} \mathrm{C}$ could mainly lead to the other shaped $\mathrm{BN}$ products such as flakes and grains, in spite of the yield which was increased.

The reaction time had an essential influence on the yield of $\mathrm{BN}$ nanotubes. In the optimum temperature range, if annealing time is less than $3 \mathrm{~h}$, the reaction will become very incomplete, and as a result, the yield will be approximately less than 30 percent.

High selectivity and high yield of the $\mathrm{BN}$ nanotubes production could be obtained when boron ratio varies within a certain range of 25 to $35 \mathrm{wt} \%$. The selectivity of the $\mathrm{BN}$ nanotubes will reach up to the highest value (about $95 \mathrm{wt} \%$ ) if boron ratio is $30.5 \mathrm{wt} \%$ for the porous precursor $\mathrm{B}_{18} \mathrm{Ca}_{2}(\mathrm{MgO})_{9}$, which was prepared by adding $33 \mathrm{wt} \% \mathrm{CaB}_{6}$. It is likely that the porous precursor has an optimum porous structure and highly dispersion state of metal catalyst and the reactant. If the boron ratio is below $25 \mathrm{wt} \%$ or above $35 \mathrm{wt} \%$, both selectivity and the yield of BN nanotubes will decrease.

\section{Conclusions}

In summary, we report a convenient and efficient method to prepare bulk quantity of boron nitride nanotubes with the diameter of 30-200 $\mathrm{nm}$ and the average length of more than $10 \mu \mathrm{m}$ by annealing a porous precursor at $1150^{\circ} \mathrm{C}$ for $6 \mathrm{~h}$ in flowing $\mathrm{NH}_{3}$. The porous precursor was produced by SHS method via heating the mixture of starting materials of $\mathrm{Mg}$, $\mathrm{B}_{2} \mathrm{O}_{3}$, and $\mathrm{CaB}_{6}$ powders to $750^{\circ} \mathrm{C}$ for 10 minutes. A base growth mechanism was proposed. The existence of metal liquid drop played an important role during the growth process of BN nanotubes. The present work demonstrates that the SHS-annealing method is effective to synthesize quantities of BN nanotubes. 


\section{Acknowledgment}

The authors acknowledge the financial support from the government of Hubei province of the China for this research work.

\section{References}

[1] D. Golberg, Y. Bando, C. Tang, and C. Zni, "Boron nitride nanotubes," Advanced Materials, vol. 19, no. 18, pp. 24132432, 2007.

[2] W. Mickelson, S. Aloni, W. Q. Han, J. Cumings, and A. Zettl, "Packing $\mathrm{C}_{60}$ in boron nitride nanotubes," Science, vol. 300, no. 5618, pp. 467-469, 2003.

[3] W. Q. Man, C. W. Chang, and A. Zettl, "Encapsulation of onedimensional potassium halide crystals within BN nanotubes," Nano Letters, vol. 4, no. 7, pp. 1355-1357, 2004.

[4] N. Koi, T. Oku, and M. Nishijima, "Fe nanowire encapsulated in boron nitride nanotubes," Solid State Communications, vol. 136, no. 6, pp. 342-345, 2005.

[5] D. Golberg, Y. Bando, Y. Huang et al., "Boron nitride nanotubes and nanosheets," ACS Nano, vol. 4, no. 6, pp. 2979 2993, 2010.

[6] R. Arenal, O. Stephan, J. L. Cochon, and A. Loiseau, "Rootgrowth mechanism for single-walled boron nitride nanotubes in laser vaporization technique," Journal of the American Chemical Society, vol. 129, no. 51, pp. 16183-16189, 2007.

[7] L. Guo and R. N. Singh, "Catalytic growth of boron nitride nanotubes using gas precursors," Physica E, vol. 41, no. 3, pp. 448-453, 2009.

[8] C. Y. Su, W. Y. Chu, Z. Y. Juang et al., "Large-scale synthesis of boron nitride nanotubes with iron-supported catalysts," Journal of Physical Chemistry C, vol. 113, no. 33, pp. 14732 14738, 2009.

[9] O. R. Lourie, C. R. Jones, B. M. Bartlett, P. C. Gibbons, R. S. Ruoff, and W. E. Buhro, "CVD growth of boron nitride nanotubes," Chemistry of Materials, vol. 12, no. 7, pp. 18081810, 2000.

[10] M. J. Kim, S. Chatterjee, S. M. Kim et al., "Double-walled boron nitride nanotubes grown by floating catalyst chemical vapor deposition," Nano Letters, vol. 8, no. 10, pp. 3298-3302, 2008.

[11] F. H. Lin, C. K. Hsu, T. P. Tang, P. L. Kang, and F. F. Yang, "Thermal-heating CVD synthesis of BN nanotubes from trimethyl borate and nitrogen gas," Materials Chemistry and Physics, vol. 107, no. 1, pp. 115-121, 2008.

[12] C. C. Tang, M. Lamy de la Chapelle, P. Li, Y. M. Liu, H. Y. Dang, and S. S. Fan, "Catalytic growth of nanotube and nanobamboo structures of boron nitride," Chemical Physics Letters, vol. 342, no. 5-6, pp. 492-496, 2001.

[13] N. Koi, T. Oku, and M. Nishijima, "Fe nanowire encapsulated in boron nitride nanotubes," Solid State Communications, vol. 136, no. 6, pp. 342-345, 2005.

[14] J. Zhang, C. Lu, and Z. Li, "Synthesis of boron nitride nanotubes by an oxide-assisted growth method," Chinese Journal of Chemical Physics, vol. 18, no. 1, pp. 113-116, 2005.

[15] Z. G. Chen, J. Zou, F. Li et al., "Growth of magnetic yardglass shaped boron nitride nanotubes with periodic iron nanoparticles," Advanced Functional Materials, vol. 17, no. 16, pp. 3371-3376, 2007.

[16] H. Tokoro, S. Fujii, and T. Oku, "Synthesis of boron nitride and carbon nanomaterials through a solid phase reduction process," Materials Chemistry and Physics, vol. 114, no. 1, pp. 204-212, 2009.

[17] Z. W. Gan, X. X. Ding, Z. X. Huang et al., "Growth of boron nitride nanotube film in situ," Applied Physics A, vol. 81, no. 3, pp. 527-529, 2005.

[18] G. Wen, T. Zhang, X. X. Huang, B. Zhong, X. D. Zhang, and H. M. Yu, "Synthesis of bulk quantity BN nanotubes with uniform morphology," Scripta Materialia, vol. 62, no. 1, pp. 25-28, 2010.

[19] K. P. Loh, M. Lin, M. Yeadon, C. Boothroyd, and Z. Hu, "Growth of boron nitride nanotubes and iron nanowires from the liquid flow of FeB nanoparticles," Chemical Physics Letters, vol. 387, no. 1-3, pp. 40-46, 2004.

[20] J. J. Fu, Y. N. Lu, H. Xu et al., "The synthesis of boron nitride nanotubes by an extended vapour-liquid-solid method," Nanotechnology, vol. 15, no. 7, pp. 727-730, 2004.

[21] J. Zhang, Z. Li, and J. Xu, "Formation and structure of boron nitride nanotubes," Journal of Materials Science and Technology, vol. 21, no. 1, pp. 128-130, 2005.

[22] Y. Chen, J. F. Gerald, J. S. Williams, and P. Willis, "Mechanochemical synthesis of boron nitride nanotubes," Materials Science Forum, vol. 312, pp. 173-178, 1999.

[23] S. Y. Bae, H. W. Seo, J. Park, Y. S. Choi, J. C. Park, and S. Y. Lee, "Boron nitride nanotubes synthesized in the temperature range 1000-1200 C," Chemical Physics Letters, vol. 374, no. 56, pp. 534-541, 2003.

[24] F. Ji, C. Cao, H. Xu, and Z. Yang, "Mechanosynthesis of boron nitride nanotubes," Chinese Journal of Chemical Engineering, vol. 14, no. 3, pp. 389-393, 2006.

[25] Y. Li, J. Zhou, K. Zhao, S. Tung, and E. Schneider, "Synthesis of boron nitride nanotubes from boron oxide by ball milling and annealing process," Materials Letters, vol. 63, no. 20, pp. 1733-1736, 2009.

[26] Y. Chen, L. T. Chadderton, J. F. Gerald, and J. S. Williams, "A solid-state process for formation of boron nitride nanotubes," Applied Physics Letters, vol. 74, no. 20, pp. 2960-2962, 1999.

[27] J. J. Velázquez-Salazar, E. Muñoz-Sandoval, J. M. RomoHerrera et al., "Synthesis and state of art characterization of BN bamboo-like nanotubes: evidence of a root growth mechanism catalyzed by Fe," Chemical Physics Letters, vol. 416, no. 4-6, pp. 342-348, 2005.

[28] C. H. Lee, M. Xie, V. Kayastha, J. Wang, and Y. K. Yap, "Patterned growth of boron nitride nanotubes by catalytic chemical vapor deposition," Chemistry of Materials, vol. 22, no. 5, pp. 1782-1787, 2010.

[29] R. Arenal, A. C. Ferrari, S. Reich et al., "Raman spectroscopy of single-wall boron nitride nanotubes," Nano Letters, vol. 6, no. 8, pp. 1812-1816, 2006.

[30] R. S. Wagner and W. C. Ellis, "Vapor-liquid-solid mechanism of single crystal growth," Applied Physics Letters, vol. 4, no. 5, pp. 89-90, 1964.

[31] T. J. Trentler, K. M. Hickman, S. C. Goel, A. M. Viano, P. C. Gibbons, and W. E. Buhro, "Solution-liquid-solid growth of crystalline III-V semiconductors: an analogy to vapor-liquidsolid growth," Science, vol. 270, no. 5243, pp. 1791-1794, 1995. 

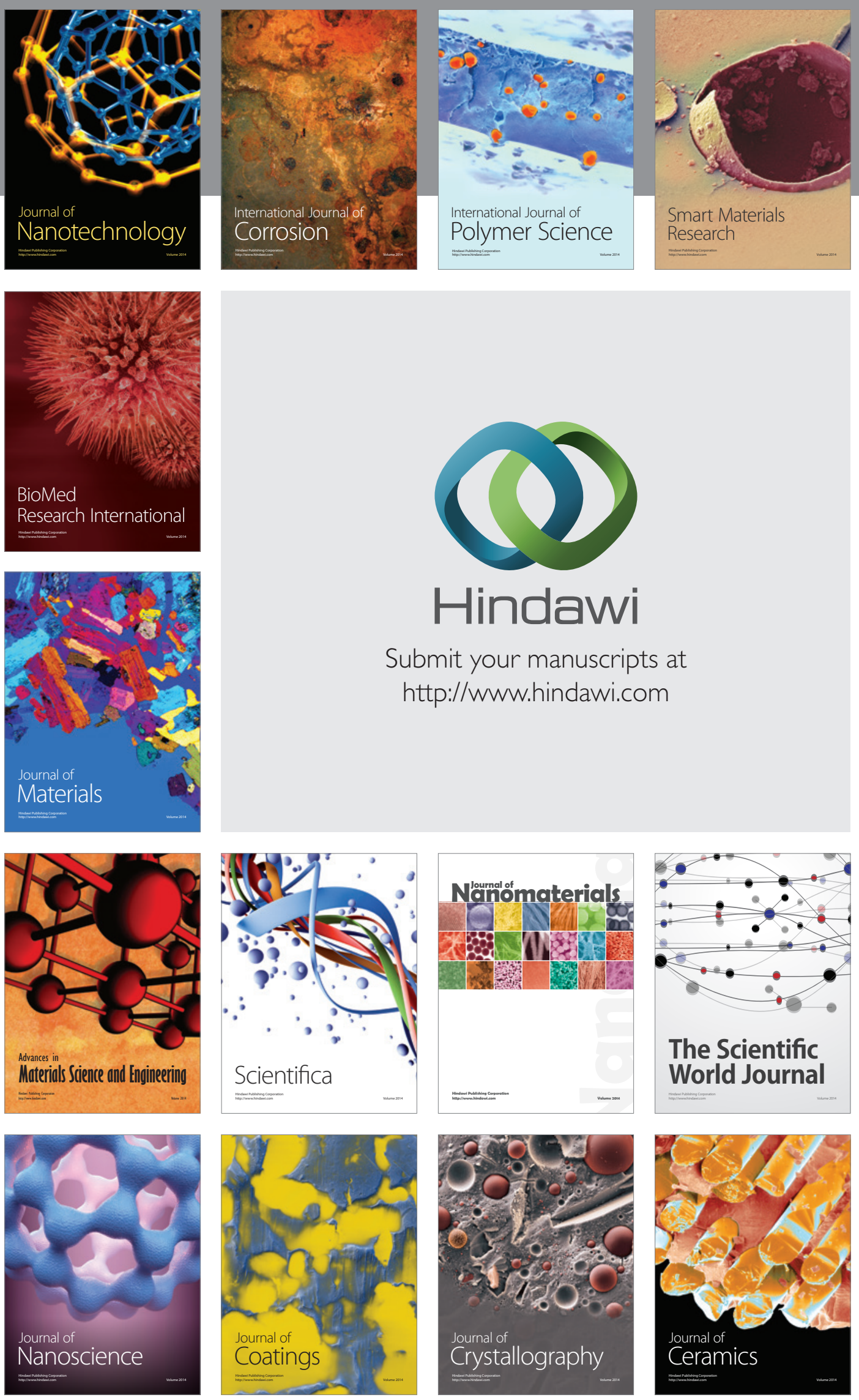

The Scientific World Journal

Submit your manuscripts at

http://www.hindawi.com

\section{World Journal}

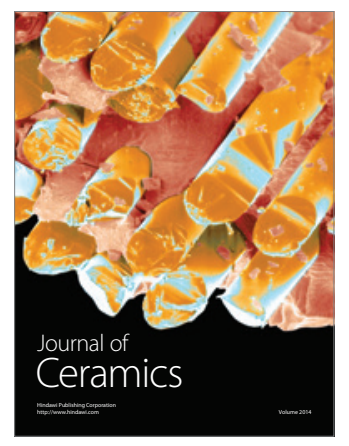

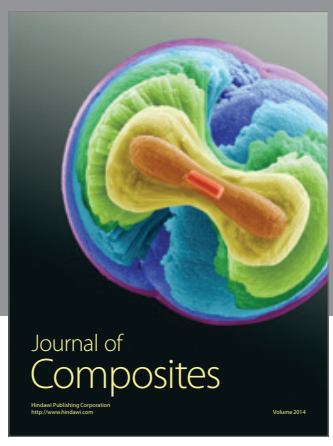
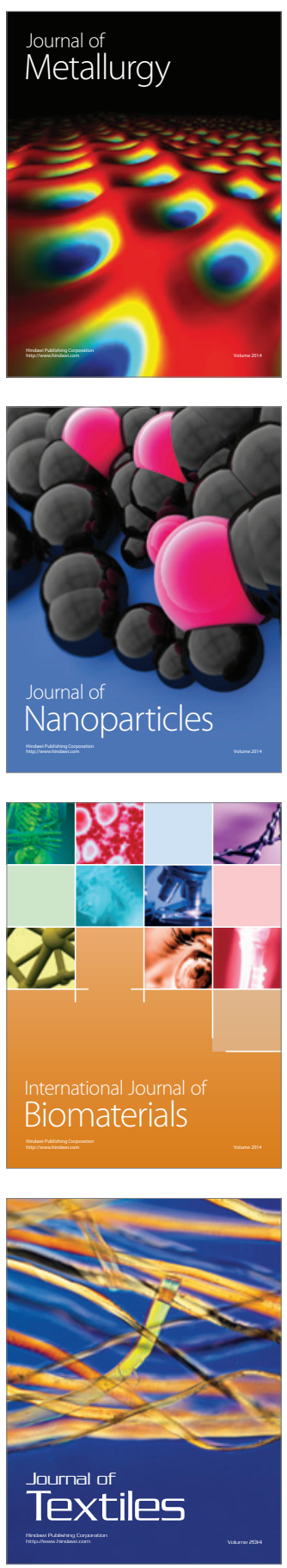